\title{
Intraocular Pressure Fluctuation - Assess in Clinical Practice
}

\author{
Marcelo Hatanaka
}

Head, Glaucoma Service, University of Sao Paulo School of Medicine, Sao Paulo, Brazil

\begin{abstract}
Intraocular pressure (IOP) is the main risk factor for the development and progression of glaucoma. Treatment is based on IOP reduction and isolated tonometric readings are usually performed for clinical management of the disease. However, IOP is not a static parameter and varies considerably throughout the day. This variability, or fluctuation, has classically been considered a risk factor for glaucoma. Nevertheless, recent studies demonstrate that pressure peaks are the most relevant IOP parameter for the prediction of visual field progression. The lack of a standard definition of IOP fluctuation compromises its clinical assessment and may explain in part the contradictory results found in the literature. This review will analyse options for IOP fluctuation assessment, their limitations and the impact of this parameter on the management of glaucoma.
\end{abstract}

\section{Keywords}

Ocular hypertension, glaucoma, intraocular pressure, IOP fluctuation, mean IOP, peak IOP

Disclosure: The author has no conflicts of interest to declare.

Received: 4 January 2013 Accepted: 23 January 2013 Citation: European Ophthalmic Review, 2013;7(1):7-9 DOI: 10.17925/EOR.2013.07.01.7

Correspondence: Marcelo Hatanaka, Av. Caxingui, 230 - Butanta, Sao Paulo - SP, 05579-000, Brazil. E: marcelohatanaka@gmail.com

Elevated intraocular pressure (IOP) is a major risk factor for the development and progression of glaucomatous neuropathy. ${ }^{1-3}$ Thus, treatment options focus mainly on IOP lowering to prevent or slower worsening of the disease. Furthermore, previous clinical trials have demonstrated the need of additional levels of IOP reduction the more advanced the stage of the disease. ${ }^{1,4-6}$

Once treatment has been initiated, follow up is usually performed with isolated IOP measurements during regular clinic hours. However, these values are just a small sample of the whole circadian variation of IOP, which is known to occur in both glaucomatous and healthy eyes. ${ }^{7}$ This may explain in part why some patients present glaucomatous progression when isolated IOP measurements suggest adequate pressure control.

The importance of a more complete IOP evaluation was demonstrated in 1963 by Drance. ${ }^{8}$ In his sample, one-third of patients with IOP measurements performed at office hours presented peaks only detected during a 24-hour diurnal tension curve (DTC). The clinical application of this information is demonstrated by Barkana et al., ${ }^{9}$ who showed that IOP evaluation performed during 24 hours was capable of revealing higher IOP levels than office hours measurements in $62 \%$ of their evaluated patients. These pressure levels were high enough to require immediate treatment change in $36 \%$ of their sample. These results emphasise the importance of 24-hour IOP monitoring despite being a time- and resource-consuming test not always feasible in the routine practice.

The main objective of a 24-hour DTC is to collect as much information as possible regarding the pressure profile of a given patient. In theory, this complete evaluation will reveal the peak, lowest and mean IOP values, as well as fluctuation within this day.
While some studies state that IOP fluctuation plays a role in glaucoma progression or development, ${ }^{10,11}$ others do not share the same findings. ${ }^{12,13}$ The lack of a standard definition and calculation of IOP fluctuation may be responsible in part for these contradictory results and also imposes difficulties to better assess this parameter in clinical practice.

It is important to mention that IOP fluctuation may reflect a short-term variability within hours or days or a long-term variability occurring between months or even years. ${ }^{14}$ Not only is the term not standardised; but also the IOP fluctuation may be calculated as the difference between maximum and minimum IOP values or as the standard deviation (SD) of the mean IOP within a given period of time.

\section{Intraocular Pressure Fluctuation Assessment and Limitations}

Short-term IOP fluctuation may be assessed as the difference between maximum and minimum IOP levels detected during a pressure curve. This method has been previously used to compare IOP fluctuation between different treatment modalities. ${ }^{15-17}$ However, measured fluctuation may not reflect the real variability through the day if IOP measurements are performed only during office hours, since nocturnal spikes may be missed. ${ }^{7-9}$

On the other hand, the use of peak and trough values to calculate fluctuation is subject to the inclusion of outliers. This could be avoided by estimating fluctuation using the SD of multiple IOP measurements, which may also be difficult to perform in clinical practice. In this case, the ideal number of visits and measurements is still unknown. Furthermore, depending on the time interval between visits, this assessment will approach long-term fluctuation definition.

In order to be clinically applicable, a parameter must be consistent and reproducible. Our research group evaluated the reproducibility 
of fluctuation during a modified DTC (MDTC, four measurements during office hours, from 08:00 AM to 04:00 PM) in patients with ocular hypertension or open angle glaucoma not under treatment on 2 consecutive days. ${ }^{18}$ In this study, fluctuation was measured as the difference between maximum and minimum values and also as the SD of all MDTC measurements. Reproducibility in both cases was considered fair. The short-term repeatability of diurnal IOP patterns one week apart in glaucomatous individuals under treatment was evaluated by Realini et al. ${ }^{19}$ The authors concluded that treated glaucomatous patients present a non-repeatable diurnal (from 08:00 AM to 08:00 PM) IOP pattern and that, as a consequence, measurement of single-day IOP variation does not characterise short-term IOP variation.

Fluctuation assessed by the water drinking test (WDT) has also been analysed. Susanna et al. ${ }^{20}$ demonstrated that eyes with worse visual field (VF) defects present higher IOP peaks and fluctuation during this test. Fluctuation was defined as the difference between pressure peak after water ingestion and baseline IOP. However, in a posterior analysis, the authors demonstrated that fluctuation during the WDT presented fair reproducibility, whereas peak IOP depicted by this test had an excellent intraclass correlation co-efficient value in patients with ocular hypertension or open angle glaucoma without treatment. ${ }^{21}$

Given these limitations regarding clinical IOP fluctuation assessment, much is expected from the development of a continuous 24 hour monitoring with a contact lens sensor. The Sensimed Triggerfish CLS (Sensimed AG) is currently under evaluation. ${ }^{22} \mathrm{~A}$ recorded IOP pattern with the device was associated with fair to good reproducibility parameters. ${ }^{23}$ However, the clinical application of these values has yet to be analysed and validated.

\section{Intraocular Pressure Fluctuation as a Risk Factor for Glaucoma}

Intraocular pressure fluctuation has classically been described as a risk factor for glaucomatous disease development and progression. Asrani et al. $^{10}$ evaluated 64 patients who performed home tonometry with a self-tonometer. Baseline office IOP presented no predictive value for progression whereas IOP range over multiple days was considered a significant risk factor (relative hazard ratio, 5.76).

More recently, Hong et al. ${ }^{24}$ demonstrated in a long follow-up (>9 years) retrospective study that eyes with lower long-term fluctuation $(S D<2)$ presented better preservation of the VF in comparison to the control group with higher fluctuation levels.

In the Advanced Glaucoma Intervention Study (AGIS), ${ }^{11}$ Iong-term IOP fluctuations, calculated as the SD of all IOP measurements during follow-up, were found to be associated with VF progression. In their analysis, eyes with IOP fluctuation $<3 \mathrm{mmHg}$ remained stable. The Early Manifest Glaucoma Treatment Trial (EGMT) presented contradictory results. ${ }^{12}$ With a median follow-up time of 8 years, $68 \%$ of the patients progressed. Mean IOP was a significant risk factor for progression (hazard ratio, 1.11), whereas IOP fluctuation was not related to glaucomatous progression. Differences in the stage of the disease between these two studies, in the inclusion and exclusion criteria as well as in the predetermined endpoints, may account in part for the discrepancy. However, it is also important to consider that in the AGIS, fluctuation assessment included IOP measurements obtained after progression occurred. A more intensive post-progression treatment would possibly have further lowered IOP with consequent increase in fluctuation. On the other side, only measurements obtained until progression detection were included for analysis in the EMGT.

To further elucidate these differences, Caprioli and Coleman ${ }^{25}$ re-analysed the data from the AGIS including only IOPS after surgical intervention until the time of the first VF deterioration and eyes with only one surgical intervention. Mean follow-up time was 7.2 years with VF progression detection in $26 \%$ of the eyes. Terciles of mean IOP were calculated and fluctuation for each group was analysed. In this model, long-term IOP fluctuation was associated with VF progression in patients with low mean IOP. The same was not found in patients with high mean IOP. A weak correlation was found between mean IOP and fluctuation. The authors hypothesise that eyes under different steady conditions such as lower or higher mean IOPs would present different responses to a sequence of stresses caused by the long-term fluctuation of IOP.

Orzalesi26 proposed an alternate explanation of the influence of IOP fluctuation over glaucomatous damage progression. Higher fluctuations levels would expose patients to a risk zone for progression, which, in other words, would be "higher- than-endurable IOP values". These high IOP levels, in fact, resemble the concept of IOP peaks.

There is an intrinsic relationship between all measured IOP parameters. The presence of isolated peaks during longitudinal data collection will elevate the mean and also the SD. Also, the occurrence of pressure peaks will elevate fluctuation measured as the difference between maximum and minimum values, specially in patients with lower mean IOPS. Despite these relationships, our study group also demonstrated that IOP peaks are much better reproducible parameters during the mDTC and WDT in comparison to fluctuation assessment. 18,21

In this context it is important to mention two large recent studies that have demonstrated that IOP peaks are better predictors of glaucomatous progression than mean IOP or fluctuation. ${ }^{27,28}$

De Moraes et al. ${ }^{27}$ retrospectively evaluated 587 eyes of 587 treated glaucomatous patients. Mean follow-up time was 6.4 years with a mean VF progression rate of $-0.45 \mathrm{~dB} /$ year for the entire population. The univariate model identified older age, exfoliation, decreased central corneal thickness (CCT), detected disc haemorrhage, presence of beta-zone parapapillary atrophy and all IOP parameters (mean, peak and fluctuation) as risk factors for VF progression. Among IOP parameters, peak and mean IOP presented the strongest association. However, in the multivariable model, only peak IOP, thinner CCT, detected disc haemorrhage and beta-zone atrophy were associated with progression. A pressure peak higher than $18 \mathrm{mmHg}$ increased the risk of progression by $81 \%$ in this study.

Gardiner et al. ${ }^{28}$ analysed predictive factors for functional progression in early and suspected glaucoma over a sequence of six visits. Evaluated baseline variables were perimetric and confocal scanning laser ophthalmoscopy parameters, IOP, age and change in visual acuity. Larger optic cup and more damaged VF were found to be predictive of faster sensitivity loss. Although there was a high correlation between maximum and mean IOP recorded over six visits, peaks were significantly predictive of progression and not mean IOP.

The classic paradigm of IOP fluctuation as a risk factor for glaucomatous progression is being questioned. Difficulties related to IOP variability 
assessment in clinical practice as well as the lack of standard definition of this parameter may me responsible in part for the contradictory results found in the literature. Twenty-four hour telemetry to fully characterise $\mathrm{IOP}$ and its variability is a promising and desirable option in glaucoma management. However, further studies are still necessary. Meanwhile, regarding IOP parameters, clinicians should focus on recording as much information as possible in order to identify and monitor those patients at higher risk for developing disease progression
1. The Advanced Glaucoma Intervention Study (AGIS): 7 The relationship between control of intraocular pressure and visual field deterioration. The AGIS Investigators, $A m\rfloor$ Ophthalmol, 2000;130(4):429-40.

2. Gordon MO, Beiser JA, Brandt JD, et al., The Ocular Hypertension Treatment Study: baseline factors that predict the onset of primary open-angle glaucoma, Arch Ophthalmol 2002;120(6):714-20; discussion 829-30.

3. Leske MC, Heijl A, Hyman L, et al., Predictors of longterm progression in the early manifest glaucoma trial Ophthalmology, 2007;114(11):1965-72.

4. Kass MA, Heuer DK, Higginbotham EJ, et al., The Ocular Hypertension Treatment Study: a randomized trial determines that topical ocular hypotensive medication delays or prevents the onset of primany open-angle glaucoma, Arch Ophthalmo 2002:120(6):701-13; discussion 829-30.

5. The effectiveness of intraocular pressure reduction in the treat . 1998;126(4):498-505

6. Mao LK, Stewart WC, Shields MB, Correlation between intraocular pressure control and progressive glaucomatous damage in primary open-angle glaucoma, Am J Ophthalmol, 1991;111(1):51-5.

7. Liu JH, Zhang X, Kripke DF, Weinreb RN, Twenty-fourhour intraocular pressure pattern associated with early glaucomatous changes, Invest Ophthalmol Vis Sci, 2003;44(4):1586-90

8. Drance SM, Diurnal Variation of Intraocular Pressure in Treated Glaucoma. Significance in Patients with Chronic Simple Glaucoma, Arch Ophthalmol, 1963:70:302-11.

9. Barkana Y, Anis S, Liebmann J, et al., Clinical utility of intraocular pressure monitoring outside of normal office hours in patients with glaucoma, Arch Ophthalmol,
2006:124(6):793-7.

10. Asrani S, Zeimer R, Wilensky 」, Gieser D, Vitale S, Lindenmuth K. Large diurnal fluctuations in intraocular pressure are an independent risk factor in patients with glaucoma, Glaucoma, 2000;9(2):134-42.

11. Nouri-Mahdavi K, Hoffman D, Coleman AL, et al., Predictive factors for glaucomatous visual field progression in the Advanced Glaucoma Intervention Study, Ophthalmology, 2004;111(9):1627-35

12. Bengtsson B, Leske MC, Hyman L, Heijl A, Fluctuation of intraocular pressure and glaucoma progression in the early manifest glaucoma trial, Ophthalmology, 2007:114(2):205-9.

13. Medeiros FA, Weinreb RN, Zangwill LM, et al., Long-term intraocular pressure fluctuations and risk of conversion from ocular hypertension to glaucoma, Ophthalmology 2008:115(6):934-40. PMCID: 2848165

14. Caprioli Intraocular pressure fluctuation an independent risk Caprioli J, intraocular pressure fluctuation: an independent risk Konstas AG, Topouzis F, Leliopoulou O, et al., 24-hour intraocular pressure control with maximum medical therapy compared with surgery in patients with advanced open-angle glaucoma, Ophthalmology, 2006:113(5):761-5 e1.

Medeiros FA, Pinheiro A, Moura FC, et al., Intraocula pressure fluctuations in medical versus surgically treated glaucomatous patients, J Ocul Pharmacol Ther 2002;18(6):489-98.

17. Wilensky JT, Zeimer RC, Gieser DK, Kaplan BH, The effects of glaucoma filtering surgery on the variability of diurnal intraocular pressure, Trans Am Ophthalmol SOC, 1994;92:377-81; discussion 81-3. PMCID: 1298517.

18. Hatanaka M, Babic M, Susanna R, Jr, Reproducibility of the Mean, Fluctuation, and IOP Peak in the Diurnal Tension Curve, J Glaucoma, 2012: [Epub ahead of print].

19. Realini T, Weinreb RN, Wisniewski S, Short-term repeatability of diurnal intraocular pressure patterns in glaucomatous individuals, Ophthalmology, 2011;118(1):47-51.

20. Susanna R, Jr Hatanaka M, Vessani RM et al, Correlation of asymmetric glaucomatous visual field damage and water-drinking test response, Invest Ophthalmol Vis Sci, 2006;47(2):641-4.

21. Hatanaka M, Alencar LM, De Moraes CG, Susanna R, Reproducibility of intraocular pressure peak and fluctuation of the water drinking test, Clin Experiment Ophthalmol, 2012; [Epub ahead of print].

22. Mansouri K, Liu JH, Weinreb RN, et al., Analysis of continuous 24-hour intraocular pressure patterns in glaucoma, Invest. ophthalmol Vis Sci, 2012;53(13):8050-6.

23. Mansouri K, Medeiros FA, Tafreshi A, Weinreb RN, Continuous 24-Hour Monitoring of Intraocular Pressure Patterns With a Contact Lens Sensor: Safety, Tolerability, and Reproducibility in Patients With Glaucoma, Arch Ophthalmol, 2012:1-6.

24. Hong S, Kim CY, Seong GJ, Long-term intraocular pressure fluctuation and visual field progression in glaucoma patients with low intraocular pressure after post-trabeculectomy with low intraocular pressure after post-trabeculectomy
phacoemulsification, J Ocul Pharmacol Ther, 2007;23(6):571-6. Caprioli J, Coleman AL, Intraocular pressure fluctuation a risk Caprioli J, Coleman AL, Intraocular pressure fluctuation a risk
factor for visual field progression at low intraocular pressures in the advanced glaucoma intervention study, Ophthalmology, 2008;115(7):1123-9 e3.

26. Orzalesi N, Fogagnolo P, Rossetti L, Intraocular pressure fluctuations in glaucoma, Arch Ophthalmol, 2008;126(5):745; author reply

27. De Moraes CG, Juthani VJ, Liebmann JM, et al., Risk factors for visual field progression in treated glaucoma, $\mathrm{Arch}$ Ophthalmol, 2011;129(5):562-8.

28. Gardiner SK, Johnson CA, Demirel S, Factors predicting the rate of functional progression in early and suspected the rate or functional progression in early and suspected 